\title{
Púrpura trombopénico idiopático crónico de la infancia: Evolución y tratamiento, 25 años de experiencia
}

\author{
Ernesto Ríos L. 1; Mirta Cavieres A.'; Patricia Dal Borgo A.'; \\ Rosario Silva C. ${ }^{1}$; Waltraud Schuh O. ${ }^{1}$
}

\section{Chronic idiopatic thrombocytopenic purpura}

\begin{abstract}
Thirly nine pedialric palients with chronic idiopathic thrombocytopenic purpura have been lollowed by the same medical team of a universily associaled children's hospital from Sartiago. Chile, for a tolal of 25 ycars. Patienrs wele treated with a slep ty step protocol that included observation, prednisone, splenectomy and immunosuppressors. Twonty three polients $15 \% \%$ eventualiy recovered, $48 \%$ did it spontoncousiy. $17 \%$ oflet prednisone seatment, $3: \%$ ofler spleneciomy and $4 \%$ after all precedent methods and immunosuppressor drugs healment. The therapeutical decissions werc alwoys laken deponding on the clinical evolution and nol aftor preslablished time schedules.
\end{abstract}

(Key words: purpura, thrombocylopenic, idiopalhic, chronic, prednisone, splenectomy, immunosuppressor therapy!.

El púrpura tsombopénico idiopálico (PT), es relativamente frecuente en la infancia. En los pacientes existen anticuerpos antiplaquetarios de tipo autoinmune, que llevan a destrucción de las plaquetas, trombopenia y hemorragia manifiesta ${ }^{1}$. El diagnóstico se fundamenta, en ausencia de otra afección, por trombopenia en la sangre periférica y mielograma normal en sus líneas celulares, excepto que puede haber hiperplasia megacarocilica. En el examen físico sólo se encuentran signos hemorrágicos sin visccromegalia ${ }^{2}$. En el PTI existe aumento de la producción de plaquetas, cuya sobrevida es muy corta cn sangre pcriférica, lo que se expresa en rápido recambio de ellas al medirlo con $\mathrm{Cr} 51$. Los macrófagos deJ sistema reticuloendotelial, preferentemente del hígado y del bazo, reconocen como anormaIes las plaquetas cubiertas de anticuerpos y las destruycn selectivamente ${ }^{3,4}$.

En el tratamiento de la afección se consideran varias ctapas. En ud mayoría de los pacientes la cvolución es autolimitada y cl trastorno cede al terninar el fenómeno autoinmune, por lo tanto,

1. Unidad de Hematología, Hospital Luis Calvo Mackenna y Departamento de Pediatría, Facultad de Mcdicina, Universidad de Chile. en ellos la conducta es observar la cvolución ${ }^{5}$. Otros pacientcs pueden requerir tratamiento con prednisona, con el múltiple objetivo de usar sus propiedades inmunosupresoras, posiblemente disminuir las inmunoglobulinas en la membrana plaquelaria e interferir con la remoción por el sistema reticuloendotelial ${ }^{6}$. En el caso de cmergencia por hemorragia o de PTI crónico se pucden usar, además, formas más drásticas de tratamiento como esplenectomia y drogas inmunosupresoras de mayor potencia ${ }^{7,8}$. En el caso de la esplencctomía el objetivo es eliminar el órgano principal de destrucción plaquetaria ${ }^{4}$, a la vez que productor de anticucrpos. También, desde hace algún tiempo, se usa gamaglobulina endovenosa en altas dosis, que produciría su efecto por bloqueo de los macrófagos, impidiendo así la destrucción de las plaquetas rodeadas de anticuerpos. el que, sin embargo, cs generalmente transitorio?.

Alrededor de $70 \%$ u $80 \%$ de los PTI evolucionan hacia la mejoría, en plazos de uno, tres y hasta seis meses. En una proporción menor de pacientes se prolonga la enfermedad más de seis meses, casos que se considcran de PTI crónico ${ }^{2}$.

El propósito de esta comunicación es describir la evolución y la respucsta al tratamiento de cuarenta pacientes con PTI crónico observados a lo largo de un período de 25 años. 


\section{Material y Métodos}

Se revisaron los antecedentes clínicos de 40 pacientes con PTI crónico registrados entre 196 ? y 1992, iodos los cuales fueron controlados por los mismos médicos, siguiendo un protocelo unifome al que ingresaron por Prr agudo. pcro la trombocitopenia se prolongó por más de scis meses, a) cabo de los cuales se efecubó una reevahuación que incluyó: hernograma, recuento de plaquetas indiecto en el frontis, VHS, mielograma, células de lupus (3 mucstras), factor reumatoideo, anticuerpos antinuclearcs, prueba de Coonbs directa y examen de orina completo.

El seguimiento de los pacientes se efcctuó mensualmente entre los 6 y 12 mescs de evolución, cada 2 meses entre los 12 y 24 meses de evolución y cada 3 meses más allá de los 24 mescs de evolución. En cada control se realizó hemograma y recuento de plaquelas. Los pacientes que se recuperaton fueron conlrojlados por 24 meses después de mejorar, consi. derándose como tales los que mostraron recuentos plaquetarios $>150000 / \mu \mathrm{l}$ en forma continua. Algunos pacientes tuvieron periodos en que alternaban recuentos de plaquelas nomales con otros de $<20000 / \mu$ por 3 meses o más $y$ fueron considerados no recuperados, clasificándoseles como PII crónico recurtente.

Para determinar la conducta terapéulica se consideró básicamente la cvoluctón clínica de los pacientes, siguiendo un curso progresivo que incluyó los siguientes pasos: observación de la evolución si no existían hemoragias o éktas eran leves; uso de predrisona (PDN) en los pacientes con signos hemorrágicos, en curas de $2 \mathbf{m g} \cdot \mathbf{k g} \cdot$ día durante 30 días. L.a esplenectomía sólo se planteó después de un año de evolución contada desde el diagnóslico, evităndola antes de la clad de 4 años, siempre que los súntomas hemortágicos justificaran la intervención. Todos los pacientes espleneclomizados rccibieron vacuna antincumocócica y se mantienen en profilaxis con penicilina ${ }^{10}$. El tratamiento inm unosupresor se efecluó como úluma opción en los pacientes en que nu se obtuvo respucsla a la ssplenectomía y cronológicamente después de Ia intervención. Para este úlimo se emplearon distintas drogas, incluyendo ciciosfosfamida (CFM) 3 mg $\cdot \mathrm{kg} \cdot \mathrm{dí}^{11}$; vincristina (VC) $1,5 \mathrm{mg} \cdot \mathrm{m}^{2} \cdot \operatorname{semana}$; vinbiastion (VBL) $6 \mathrm{mg} \cdot \mathrm{m}^{2}$ adosada a plaquelas por cada transfusión ${ }^{\mathrm{g}}$ y ciclosperina $10 \mathrm{mg} \cdot \mathbf{k g} \cdot$ ‘fía 12 .

Debe serialarse que durante el esindio se afinó y comnprendió mejor la enfermedad de Von Willebrand, en su variedard II B, que puede evolucionat con trombopenia crónica ${ }^{13}$. Por este motivo, a partir de 1986 , siempre que se propuso este diagnóstico diferencial se hicieron estudios complemenlarios con pruebas de laboratorio orientadar a esta enfermedad, incluyendo tiempo de sangría de Ivy ${ }^{14 .} 15$, mediciones del factor VIII coagulante ${ }^{16}$, factor Von Wi. Jebrand" ${ }^{17}$, cofactor ristocetina ${ }^{18}$ y cstudio de muluímeros del [actor VII's

\section{Resultados}

Uno de los 40 pacientes no concurrió a sus controles y fue cxcluido por no ser evaluable. Diccinueve pacientes eran de sexo masculino. La edad al diagnóstico fluctuó entre 7 meses y 15 años, siendo en $69 \%$ de los casos entre 2 y 7 años (figura 1). Todos los exámenes de laboratotio, diagnóstico de PTI crónico fucron normales, exceptuando el recuento de plaquatus.

Veintitrés pacientes (59\%) se recuperaron, de acuerdo con el critcrio terapśúco expresado. Once de cllos esponláneamente, cuatro después de usar la predisona, sietc después de esplenectomía y uno al agregar inmunosupresor. Los períodos de recuperación desde el diagnóstico, según los diversos tratamientos efectuados se describen en la tabla 1. De los once pacientes que se recuperaron de manera cspontánea, cinco eran asintomáticos y solamente fueron obscrvados, mientras los otros seis recibieron prednisona, pero sin respuesta positiva. Debe destacarse que la recuperación espontánea corresponde a $28 \%$ del toLal de la muestra y puede ocurrir hasta 60 meses después del diagnóstico. La prednisona tuvo un cfecto directo en la recupcración de cuatro pacientes, aunque todos cllos habian recibido corticoides en el período agudo del PTI sin resultados. La esplenectomía tuvo un efecto curativo en siete pacientes, todos recibicron prednisona previamente, cntre una y cinco curas, siendo postergado el procedimiento aún hasta 4 ó 5 años después del diagnóstico en los pacientes cuya evolución permítió hacerlo. Un sólo paciente de este grupo se rccuperó al agregar finalmente ciclofosfamida.

En dieciséis pacientes (41\%) no se registró recuperación. Cuatro de cllos no han recibido tratamiento, seis han sido tratados con prednisona, cinco fueron esplenectomizados sin resultados y en cuatro se uso, posteriormente, inmunosupre-

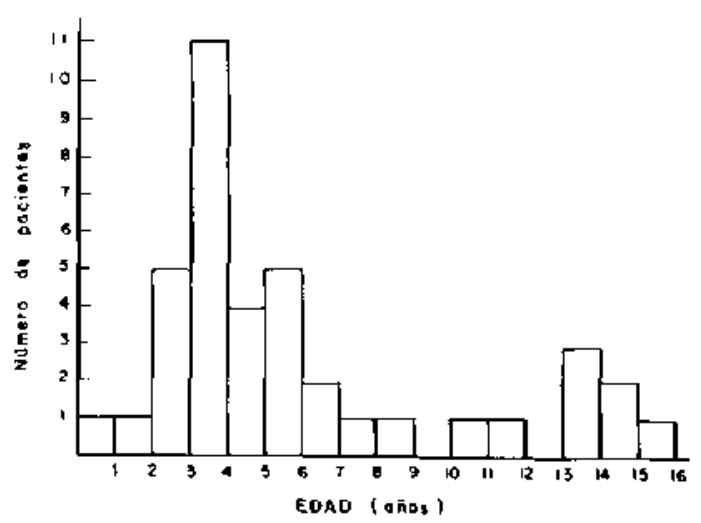

Figura: edad de diagnóstico en 39 pacjentes con PTI cronico. 
Tabla 1

Periodo de recuperación según tratamiento efectuado en 23 pacientes con púrpura trombopénico idiopátjco crónico

\begin{tabular}{|c|c|c|c|c|c|}
\hline $\begin{array}{l}\text { Tiempo de } \\
\text { recuperación desde } \\
\text { dlagnóstico } \\
\text { (meses) }\end{array}$ & $\begin{array}{c}\text { Recuperación } \\
\text { espontáne̊ }\end{array}$ & PDN & $\begin{array}{c}\text { PDN + } \\
\text { esplenectomía }\end{array}$ & $\begin{array}{l}\text { PDN + } \\
\text { esplenectomía + } \\
\text { inmunosupresor }\end{array}$ & Total \\
\hline$<12$ & 1 & - & $2^{*}$ & $1^{*}$ & 3 \\
\hline $13-24$ & 4 & 2 & $i$ & - & 7 \\
\hline $25-36$ & 3 & 1 & 3 & - & 7 \\
\hline $37-48$ & 2 & - & - & - & 2 \\
\hline $49 \cdot 60$ & 1 & 1 & 2 & - & 4 \\
\hline Total & 11 & 4 & 7 & 1 & 23 \\
\hline
\end{tabular}

* Pacienle grave que obligó a modificar el esquema terapéutico.

Tubla 2

Tiempo de evolución y tratarniento recibido en 16 pacientes con púrpura trombopénico idiopático crónico no recuperados

\begin{tabular}{|c|c|c|c|c|c|c|}
\hline $\begin{array}{l}\text { Tiempo de } \\
\text { evolución desde } \\
\text { diagnóstleo } \\
\text { (añes) }\end{array}$ & $\begin{array}{c}S \ln \\
\text { tratamiento }\end{array}$ & Pred & $\underset{\text { esplenectomia }}{\text { Pred + }}$ & $\begin{array}{c}\text { Pred + } \\
\text { esplenectomía }+ \\
\text { immunosupres }\end{array}$ & $\begin{array}{c}\text { Pred + } \\
\text { Inmunosupres }\end{array}$ & Total \\
\hline$<1$ & - & $1^{*}$ & - & - & - & 1 \\
\hline $1-2$ & - & - & - & - & - & 0 \\
\hline $2-3$ & 1 & $2^{*}$ & - & l & - & 4 \\
\hline $3-4$ & $1+$ & $1 \&$ & - & - & - & 2 \\
\hline $4-5$ & $2^{*}+$ & - & - & - & - & 2 \\
\hline $5-6$ & - & - & - & 1 & - & 1 \\
\hline $6-7$ & - & - & 1 & - & $1^{*}$ & 2 \\
\hline$>7$ & - & $2+\&$ & - & $2^{*}+$ & - & 4 \\
\hline Total & 4 & 6 & 1 & 4 & 1 & 16 \\
\hline
\end{tabular}

*: Paciente descontinuo el control. Pred: prednisona.

t: PTI crónico recurrentc, \&: familia rechazó esplenectomía.

sores. Finalmente un paciente recibió prednisona y luego inmunosupresor, saliéndose del protocolo inicial, porque los padres no aceptaron la esplenectomía. El tiempo de evolución desde el diagnóstico y el tratamiento recibido en los 16 pacientes no recuperados, se muestra en la tabla 2. El hecho de observar solamente la evolución en cuatro de los pacientes no recuperados, obedece a que no han presentado hemorragia y sus recuentos de plaquetas oscilan entre $60000 \mathrm{y}$ $110000 / \mu$; todos comenzaron como PTI agudo, en tres se realizó el estudio de von Willebrand, que resultó negativo y en el cuarto no se pudo efectuar por asistencia irregular a controles. Los seis pacientes no recupcrados y tratados exclusivamente con prednisona no continuaron en el protocolo por diferentes motivos. Tres suspendicron sus controles entre 9 meses y 3 años de 
cvolución, dos rechazaron la esplenectomía y uno ticne una cvolución crónica recurrente.

La esplenectomía, como procedimicnto terapéutico, se realizó en 14 pacientes $(36 \%)$, todos ellos recibieron previamente una a cinco curas de prednisona sin resultados, con respuesta temporal a este tratamicnto en tres, uno de los cuales recibió, además, inmunoglobulina endovenosa (400 $\mathrm{mg} \cdot \mathrm{kg} \cdot \mathrm{dia}-5 \mathrm{~d}$ dias) antes de la intervención, todo sin resultados. Sietc pacientes se recuperaron con la operación (50\%), seis no respondicron y uno no volvió a sus controles, por lo que no fuc evaluable. Uno de los pacientes que no respondió to hizo al agregar ciclofosfamida al tratamiento, como ya se relató. El momento de la esplenectomía en la evolución y sus resultados se describen en la tabla 3 y la edad de los pacientes al practicaria en la tabla 4 . Como complicación de la esplenectomía, un paciente recuperado sufrió meningitis neumocócica tres años más tarde, de la que se recuperó con secuelas, pero cinco años después de la csplencctomía presentó una scpsis ncumocócica y falleció. En ese tiempo no existía vacuna atineumococo, ni se usaba la profilaxis con penicilina.

El Iratamiento inmunosupresor se emplcó en scis enfermos (15\%), cinco habían sido esplenectomizados sin respuesta. Solamente hubo recuperación en un niño en quien se usó ciclofosfamida $3 \mathrm{mg} \cdot \mathrm{kg} \cdot$ dia a partir del segundo mes después de la espleneclomía, durante $41 / 2$ meses y fue dado de alta 4 años después de su recuperación. Los pacientes que no respondieron recibieron diferentes esquemas terapéuticos, uno ciclofosfamida por 3 meses; otro por 2 moses $y$ luego cuatro transfusiones de plaquetas con vinblastina; el otro vincristina por dos semanas y lucgo ocho transfusiones de plaquetas con vinblastina. Este último paciente inició una evolución crónica recurtente despućs de este tratamiento. EI cuarto paciente recibió ciclosporina por un año y finalmente, el último de ellos, el que se salió de protocolo inicial, no fue csplenectomizado, recibiendo vincristina durante cinco semanas. Si no se observaba recuperación del recuento de plaquetas, con cualquiera de los tratamientos efoctuados y siempre que persistieran las hemorragias, se usó prednisona en baja dosis, $5 \mathrm{mg}$ en días alternos, independiente del paso, para actuar sobre la pared capilar.

Siete pacientes cursaron con PTI crónico recurrente, tres se recuperaron en forma espontá-
Tabla 3

Momento de la esplenectomía y sus resultados en 14 pacientes con púrpura trombopénico idiopático crónico

\begin{tabular}{ccccc}
\hline $\begin{array}{c}\text { Tiempo de } \\
\text { evolucion desde } \\
\text { diagnóstico } \\
\text { (años) }\end{array}$ & $\begin{array}{c}\text { Recupe. } \\
\text { rados }\end{array}$ & $\begin{array}{c}\text { No recupe- } \\
\text { rados }\end{array}$ & $\begin{array}{c}\text { No } \\
\text { evaluable }\end{array}$ & Total \\
\hline$<1$ & $1 *$ & $1^{*}$ & - & 2 \\
$1-2$ & 1 & 3 & 1 & 5 \\
$2-3$ & 3 & 1 & - & 4 \\
$>3$ & 2 & 1 & - & 3 \\
\hline Total & 7 & 6 & 1 & 14 \\
\hline
\end{tabular}

* Pacicnte grave que obligó a modificar esquema terapéutico.

\section{Tabla 4}

Edad de pacientes al ser esplenectomizados

\begin{tabular}{cc} 
Edad (años) & $N^{9}$ pacientes \\
\hline$<4$ & $1^{*}$ \\
$4-6$ & 1 \\
$6-8$ & 3 \\
$8-10$ & 1 \\
$10-12$ & $2^{*}$ \\
$12-14$ & 1 \\
$>14$ & 14
\end{tabular}

* Paciente esplenectomizado antes de un año de cvo. lución (ver texto).

nea, el cuarto inició esa modalidad evolutiva después de esplenectomía y tratamiento inmunosupresor y los tres restantes han persistido en ella por plazos de 3 años a más de 7 años de control, recibiendo prednisona en diferentes períodos de trombopenia sin resultados.

Finalmente en forma anecdótica debe relatarse que hubo dos pacientes de sexo femenino. esplenectomizadas y no recuperadas, que hacia el final de la adolescencia (18 y 19 afios) quedaron embarazadas. En sus controles no tuvieron problemas obstétricos, recibieron conticoides en el último mes de embarazo, tuvieron partos por vía vaginal normales y ambos recién nacidos, 
afortunadamente, no presentaron trombocitopenia. En Jas dos pacientes, en el año siguiente al parto, mejoraron los niveles de plaquetas a alrededor de $100000 / \mathrm{pl}$ pero sin recuperarse totalmente.

\section{Comentario}

No cxisten hoy elementos clínicos o de laboratorio que permitan predecir la evolución del PTI, salvo esperar que el tiempo de la clasifi. cación de agudo o crónico probablemente es el mismo fenómeno inmunológico, que por causa desconocida prolonga su proceso. En todos los pacientes de este esludio la enfermedad empezó como PTI agudo y se prolongó por más de seis meses, sin que se detcctaran trombopenias secundarias a otras enfermedades hematológicas, inmunológicas o drogas. Clásicamente en el PTI crónico de los adultos se ha descrito que la enfermedad predomina cn mujeres y que la mayor frecuencia es a partir de la pubertad ${ }^{2}$, pero en esta serie no se encontraron diferencias de sexo y la mayor incidencia al diagnóstico fue entre 2 y 7 años, lo que expresa otras diferencias entre el PTT infantil y el de adultos. Es interesante destacar que la recuperaciơn espontánea puede ocurrir hasta cinco afios después del diagnóstico, terminando el fenómeno autoinmune sin causa aparente, revirtiéndose un proceso que también se inició sin una explicación etiopatogénica. De los pacientes en esta situación algunos recibieron prednisona en varias curas, sin respuesta favorable concomitante. Por otra parte, los pacientes recuperados en inmediata relación con el uso de prednisona lo hicieron hasta 5 ax̃os después del diagnóstico y habian recibido curas anteriores sin respuesta. La pregunta es por qué la prednisona a veces es capaz de controlar el proceso autoinmune y otras no. Es posible que la intensidad de este hubiese disminuido espontáncamente facilitando el efecto del corticoide, pero también cabe que, definitivamente, la enfernedad fuese en camino de terminar sola.

La intensidad del proceso autoinmune, en todos los pacientes en que debió usarse csplenectomía, fue de tal magnitud que llevó, por un lado, a una evolución clínica más tormentosa y. por otro, a ausencia de efecto de la prednisona, lo que obligó a realizar un procedimiento terapéutico más drástico. La esplenectomía sólo tuvo resul- tados positivos en $50 \%$ de los pacientes sometidos a ella, esto indica que el fenómeno autoinmunc es de mayor magnitud aún y que la destrucción de plaquetas es asumida por macrófagos del sistema reticuloendotelial ubicados en otros órganos, de preferencia el hígado ${ }^{20}$. No existe ningún cstudio clínico o de laboratorio que permita anticipar el resultado de la esplenectomía ${ }^{21}$. En varios pacientes el procedimiento no fue aceptado por los padres.

Finalmente se debe destacar el pobre resultado de los inmunosupresores, ya que sólo un paciente $(17 \%)$ respondió a su uso después de la esplenectomía. Los enfermos que no responden a ningún tipo de tratamiento, de los cuales la mayoría tiene 5 o más años de control en esta scrie, plantean una dificultad evidente en el seguimiento. Hoy existe la posibilidad de usar gamaglobulina endovenosa en altas dosis, que en esta serie no fue usada por el coste, pues un tratamiento completo vale 100 dólares por $\mathrm{kg}$ de peso del paciente, con resultados tambićn inciertos ${ }^{9}$.

El PTI crónico recurrente plantea aún más incógnitas en la etiopatogenia, porque probablemente existe una oscilación en la intensidad del proceso autoimmune, con períodos de baja actividad y normalización del recuento plaquetario.

El hecho que, entre seis pacientes con recuentos plaquetarios intermedios, cl estudio complementario de coagulación permitió identificar a uno con enfermcdad de Von Willebrand, aconseja quc, en todo paciente trombopénico en quien la iniciación de la enfermedad no sea la de un PTI agudo clásico o la trombopenia pcrsista más de 6 meses, sca efectuado el estudio de los factores de coagulación para definir el diagnóstico.

\section{Resumen}

Se presentan 39 pacientes con PTI crónico, controlados por largo tiempo por el mismo equipo médico. Se analizan los resultados obtenidos con diferentes tratamicntos, de acuerdo al scguimiento de un protocolo que incluye observación, uso de prednisona, esplenectomía y uso de inmunosupresores. Se registró recuperación en 23 pacientes $(59 \%)$, de ellos $48 \%$ lo hizo en forma espontánca, $17 \%$ en coincidencia con uso de prednisona, $31 \%$ con la esplenectomia y $4 \%$ con inmunosupresores. Se hace énfasis en que la de- 
cisión terapéutica se tomó dependiendo de la evolación clínica y no por cumplimiento de plazos predeterminados.

(Palabras clave: púrpura, trombocitopénico, idiopático, crónico, prednisona, esplencctomia, inmunosupresores).

\section{Referencias}

1. Karparkin $M$ and Karpatkin S: Immune thrombo. cytopenia in children. Am I Pedialt Hernatol Oncol 1981; 3: 213-219.

2. Waters AH: Autoimmune thrombocytopenia: clinical aspects. Seminars Hematol 1992; $29: 18-25$.

3. Aster RH: Platelet sequestration studies in man. $\mathrm{Br}_{\mathrm{J}} \mathrm{J}$ Haematol 1972; 22: 259-263.

4. Branelog I: Platelet kinetic in idiopathic thrombocytopenic purpura before and at different times after splenectomy. Br J Haematol 1975; 29: 413-426.

5. Lusher JM, Emami A, Ravindranath Y, et al.: Idjopathic thrombocytopenic purpura in children. The case for management without corticosteroid. Am I Pediatr Henalol Oncol 1984; 6: 149-157.

6. Buchoman GR: Childhood acute idiopathic thrombocytopenic purpura: how many test and how much treatment required? J Pedialt 1985: 106: 928-929.

7. Ahn YS. Harrington WJ, Seeiman RC, et al.: Vincristine therapy of idiopathic and secondary thrombocy topenias. N Engl J Med 1974: 291: 376-380.

8. Ahn YS, Byrmes JJ, Harrington WJ, et al.: The treatment of idiopathic thrombocylopenia with yinblastine-loaded platelets. N Engl J Med 1978: 1101-1107.

9. Imbach $F$. Berchtold $W$, Hirt A: Intravenous imnunoglobulin versus oral corticosteroids in acute immune thrombocylopenic purpura of childhood. Lancet 1985; 2: $464-468$.
10. Hosea SW, Brown EJ. Hamburger MI, et al.: Opsonic reguirements for intravascular clearance after splenectomy. N Engl J Med 1981; 304: 245-250.

11. Hengst $J C$, Kempf $\boldsymbol{R}$ : Immunc modulation by cyclophosphamide. Clin Immunol Aliergy 1984; 2: 199216.

12. Thomson AW. Neild GH: Cyclosporin: use outside Iransplantation. Br Med J 1991; 302; 4-5.

13. Mezzano D, Pereira J, Quiroga T: Enfermedad de Von Willebrand. Rev Med Chile 1990; $118: 320-329$.

14. Mielke CH, Koneshiro IA, Maher JM, et ai.: Nomal Ivy blecding tione and its prolongation by aspirin. Blood 1969: 34: 204-209.

15. Rlos $E$, Pinocher $M$, Mandujano $M$ : Tiempo de sangría de Ivy modificado: vatores nomales en edad pediátrica. Rev Chil Pediatr 1982; 53: 552-554.

16. Langdell RD, Wagner RH, Brinkhows KM: Effect of antihemophilic factor on one stage clouing test. I I ab Clin Med 1953; 41: 637-642.

17. Zimunerman TS, Hoyer LW, Dickson L, et al.: Determination of the Von Willebrand's disease antigen in plasma by quantitative immunoclectrophoresis. J Lab Clin Med 1975: 85: 318-328.

18. Allain $J P$, Cooper $H A$, Wagner $R H$, et al.: Platelet fixed with paraformaldehyde: a new reagent for assay of Von Willebrand factor and platelel aggegating factor. J L_ab Clin Med 1975; 85: 318-328.

19. Ruggeri ZM. Zimnerman TS: Variant Von Wilebrand disease: characterization of two subtypes, by analysis of multimeric composition of FVILI/ $\mathrm{WW}$ factor in plasma and platelets. I Clin Invest 1980; 65: 13181325.

20. Aster RH, Keene WR: Siles of pialelet destruction in idiopathic thrombocytopenic purpura. Br J Hatmatol 1969; 16: 61-71.

21. Julta $A$, Aragwas $C$, Rossello $J$, ef al.: Lack of useful clinical predictors of response to splenectomy in patients with chronic idiopathic thrombocytopenic purpura. Br $\int$ Haematol 1990: 76: 250-255. 